\title{
Boas práticas na sala de parto e ansiedade em puérperas: o que pode influenciar?
}

\section{Good practices in the delivery room and anxiety in postpartum women: what can it influence?}

Buenas prácticas en la sala de partos y ansiedad en puérperas: ¿en qué puede influir?

\section{Resumo}

Durante $\mathrm{o}$ ato de parir a mulher tem diversos sentimentos, entretanto, atualmente com a institucionalização do parto nos hospitais, não há a priorização dessas emoções e aspectos fisiológicos o que existe é o excesso de intervenções. Isso pode acarretar a falta de contato pessoal e os aspectos emocionais ficam em segundo plano. Nesse sentido, o estudo tem como objetivo identificar se as boas práticas na sala de parto, a partir dos componentes do parto humanizado, influenciam o escore de ansiedade das mulheres no pós-parto imediato. O estudo é prospectivo e transversal, com 810 puérperas, que assinaram o TCLE ou TALE, dos alojamentos conjuntos em uma maternidade filantrópica em Sergipe. Os instrumentos usados na pesquisa foram os questionários baseados em duas concepções de ansiedade. Desse modo, os fatores encontrados que influenciam na ansiedade foram o contato pele a pele, a amamentação na primeira hora pósparto, o tipo de parto, o uso das práticas não farmacológicas para alívio da dor, a posição de expulsão, a presença do acompanhante de livre escolha e um parto não violento.

Palavras-chaves: Parto humanizado; Trabalho de parto; Ansiedade-estado; Ansiedade-traço; Puerpério.

\begin{abstract}
During the act of giving birth, the woman has several feelings, however, currently with the institutionalization of childbirth in hospitals, there is no prioritization of these emotions and physiological aspects, what exists is excessive processing. This can lead to a lack of personal contact and emotional aspects take a back seat. In this sense, the study aims to identify whether good practices in the delivery room, from the components of humanized childbirth, influence the anxiety score of women in the postpartum immediately. The study is prospective and cross-sectional, with 810 postpartum women, who signed the IC or TALE, from the deactivated groups in a philanthropic maternity hospital in Sergipe. The instruments used in the research were questionnaires based on two conceptions of anxiety. Thus, the factors found to influence anxiety were skin-to-skin contact, breastfeeding in the first hour postpartum, type of delivery, use of non-pharmacological practices for pain relief, expulsion position, presence of free choice companion and a non-violent birth.
\end{abstract}

Keywords: Humanized birth; Childbirth; State anxiety; Trait anxiety; Puerperium. 


\begin{abstract}
Resumen
Durante el acto de dar a luz, la mujer tiene varios sentimientos, sin embargo, actualmente con la institucionalización del parto en los hospitales, no existe una priorización de estas emociones y aspectos fisiológicos, lo que existe es un procesamiento excesivo. Esto puede llevar a una falta de contacto personal y los aspectos emocionales pasan a un segundo plano. En este sentido, el estudio tiene como objetivo identificar si las buenas prácticas en la sala de partos, a partir de los componentes del parto humanizado, influyen en el puntaje de ansiedad de las mujeres en el posparto de manera inmediata. El estudio es prospectivo y transversal, con 810 puérperas, que firmaron el IC o TALE, de los grupos desactivados en una maternidad filantrópica de Sergipe. Los instrumentos utilizados en la investigación fueron cuestionarios basados en dos concepciones de la ansiedad. Así, los factores que influyeron en la ansiedad fueron el contacto piel con piel, la lactancia materna en la primera hora posparto, el tipo de parto, el uso de prácticas no farmacológicas para el alivio del dolor, la posición de expulsión, la presencia de acompañante de libre elección y un acompañante no violento. nacimiento.
\end{abstract}

Palabras clave: Nacimiento humanizado; Trabajo de parto; Estado de ansiedad; Rasgo de ansiedad; Puerperio.

\title{
1. Introdução
}

Durante o trabalho de parto a mulher passa por diversas sensações e sentimentos. Entretanto, atualmente com a institucionalização do parto nos hospitais, não há a priorização dessas emoções e aspectos fisiológicos, mas sim um excesso de intervenções (Mendes \& Rattner, 2020). A conquista da realização do parto hospitalizado e a criação de maternidades tiveram resultados significativos, tais como a redução de óbitos maternos e neonatais, de modo que foram sonegadas a individualidade e a autonomia das mulheres (Costa Souza, et al. 2019)

Tudo isso pode levar à falta de contato pessoal e os aspectos emocionais ficam em segundo plano. Não basta apenas criar técnicas, é preciso criar laços de relacionamento humano, pois a partir deles a mulher se sentirá mais confortável na passagem desse processo que é gestar e parir (Martins Matei, Mota de Carvalho, Henrique Silva \& Barbosa Merighi, 2003).

Questiona-se essa conduta alertando os profissionais da saúde que atendem a mulher no processo da gestação e do parto sobre seus atos. Para que percebam a provável influência do modelo econômico onde a cultura da tecnologia, da racionalização do tempo, do produzir e do consumir estejam atropelando suas condutas profissionais e, assim, intervindo excessivamente em processos naturais como é o parto (Cechin, 2002).

A proposta de humanização da assistência ao parto vem ao encontro dos anseios das mulheres de que a vivência do parto seja uma oportunidade de atuar como protagonista, fazendo com que o parto deixe de ser um evento meramente biológico, mas sim uma experiência humana, que deve ser experimentada de acordo com suas expectativas (Gonçalves, Aguiar, Mereghi, \& Jesus, 2011).

O parto é um processo normal e natural, um período vulnerável para a saúde da mulher, no qual o meio ambiente e as atividades de saúde exercem grande influência. Dessa maneira, a assistência ao parto deve ser centrada nas necessidades das mulheres, que deverão ter seus direitos à informação e à decisão informada respeitados (Macedo, Quitete, Lima, Santos \& Vargens, 2008).

Este estudo tem como objetivo identificar se as boas práticas na sala de parto a partir dos componentes do parto humanizado influenciam no escore de ansiedade nas mulheres no pós-parto imediato.

\section{Metodologia}

O trabalho trata-se de um delineamento da pesquisa intitulada "Avaliação dos componentes de ansiedade em pacientes no pós-parto imediato em uma maternidade da rede SUS em Aracaju/SE” cuja metodologia já foi descrita como um estudo prospectivo e transversal, de abordagem quantitativa, com puérperas dos alojamentos conjuntos em uma maternidade filantrópica de risco habitual em Sergipe. Essa maternidade com 91\% dos leitos destinados ao SUS, realizou 10.920 partos em 2018, sendo a média mensal de 945,3 partos. A prevalência de parto vaginal neste serviço em 2018 foi de $73 \%$. A população da amostra foi de puérperas provenientes dos 75 municípios do estado de Sergipe internadas na maternidade durante o período da coleta de dados. Para o 
tamanho da amostra utilizou-se a fórmula de Barbetta (Barbetta, 2002) considerando o número de partos realizados no serviço em 2018, com erro amostral de 5\%, resultando em 810 mulheres, dentre as quais houve 7 perdas totais. Em tal pesquisa, no período de Setembro de 2019 a Fevereiro de 2020 os pesquisadores realizaram diariamente as entrevistas e a coleta dos dados das puérperas nas primeiras 48 horas após o parto.

Ainda referente à metodologia da pesquisa, os critérios de inclusão foram: puérperas de todas as idades internadas na maternidade no período da coleta e que aceitem participar do estudo, assinando o Termo de Consentimento Livre e Esclarecido (TCLE) ou Termo de Assentimento Livre e Esclarecido (TALE). Foram excluídas da pesquisa pacientes que apresentarem história atual ou passada de depressão ou tratamento psiquiátrico, alcoolismo ou abuso de drogas, gestação gemelar, filhos estejam natimortos ou que sejam encaminhadas a Unidade de Terapias Intensiva.

Dessa forma, foram avaliados alguns fatores clínicos como, tipo de parto, opinião da paciente se o parto foi considerado "violento", se utilizou algum método não farmacológico de alívio da dor dentre eles o banho morno, massagem e exercício com a bola, se foi incentivada a andar e se movimentar durante o parto, teve acompanhante de livre escolha, recebeu dieta líquida, realizou episiotomia, teve direcionamento dos puxos da dor durante o parto, qual a posição adotada no período expulsivo, realizou contato pele a pele com o recém-nascido e amamentação na primeiras hora pós-parto.

Em sequência foram aplicados o questionário IDATE-Traço e IDATE-Estado, instrumentos validados para aferição de ansiedade nas pacientes participantes do estudo.

À respeito dos riscos e benefícios da pesquisa, por se tratar de análise do prontuário, cartão da gestante e resposta ao questionário não apresentou riscos físicos, entretanto há riscos de constrangimento, quebra de sigilo e do anonimato. Para minimizar esses riscos os pesquisadores se comprometeram a respeitar a privacidade da paciente durante a coleta de dados, permitiram que ela somente respondesse o que se sentia confortável e guardaram as informações em local seguro e restrito. Além disso, somente os pesquisadores tiveram acesso às informações que foram utilizadas exclusivamente para a realização deste estudo.

Os dados coletados na pesquisa foram armazenados em um banco de dados no Microsoft Excel 2010 e analisados por intermédio de estatística pelo programa Jasp versão 0.12.1. Desse modo, todas as variáveis nominais do questionário sociodemográfico e clínico adotaram o número (1) para "sim" e (2) para "não" a fim de converter as palavras em números para serem lidas pelo programa.

Para as análises estatísticas do questionário Idate foi necessário inverter na planilha as afirmações positivas; $1 ; 6 ; 7 ; 10 ; 13 ; 16$ e 19 no Idate-Traço e 1;2;5;8;10;11;15;16;19 e 20 no Idate-Estado, em seguida foi calculado o score e a correlação com os fatores clínicos e socioeconômicos.

A correlação entre os fatores e os níveis de ansiedade foram realizadas através do teste ANOVA duas vias seguido do pósteste de Tukey. O nível de significância adotado para todas as análises foi de 5\%.

Este trabalho foi aprovado no CEP da Universidade Tiradentes sob o parecer 3.695.763. Todas as pacientes assinaram o Termo de Consentimento Livre e Esclarecido ou o Termo de Assentimento Livre Esclarecido.

\section{Resultados}

No questionário Idate-Estado, foi identificado que o parto normal $(\mathrm{p}=0,005)$ mostrou valores menores do escore de ansiedade. Já a respeito da posição no período expulsivo $(\mathrm{p}=0,003)$, do parto considerado violento $(\mathrm{p}=0,017)$ mostrou uma pontuação maior no escore de ansiedade. Além disso, não ter um contato pele a pele $(\mathrm{p}=0,011)$ e não amamentar na primeira hora pós parto $(\mathrm{p}=0,031)$ aumentaram o escore de ansiedade.

Já no Idate-Traço o uso das práticas não farmacológicas para alívio da dor $(\mathrm{p}=0,022)$ como exercício com a bola, banho morno e massagem, posição de expulsão em decúbito lateral esquerdo $(\mathrm{p}=0,011)$ e a presença do acompanhante de livre escolha 
$(\mathrm{p}=0,013)$ tiveram impacto negativo na pontuação do Idate. Já os fatores que influenciaram no aumento do escore de ansiedade foi a dieta líquida $(\mathrm{p}=0,006)$ e posição ginecológica $(\mathrm{p}<0,001)$.

\section{Discussão}

A partir do último trimestre de gestação as gestantes já estão em um nível alto de ansiedade por conta da proximidade com o parto (Pereira RDR, et al.,2011). Entretanto, esse nível de ansiedade pode aumentar ainda mais ou podem ser minimizados.

O instrumento IDATE usado no estudo foi traduzido e validado para português por Biaggio e Natalício. O questionário se baseia em duas concepções de ansiedade conforme definidos por Spielberger: o traço e o estado. O estado de ansiedade é o estado emocional transitório ou condição do organismo humano que pode variar de intensidade e tempo caracterizado por momentos desagradáveis de tensão e apreensão conscientemente percebidos. O traço de ansiedade, por sua vez, refere-se às situações ameaçadoras geralmente influenciada por experiências passadas do indivíduo (Biaggio, et al., 1977).

No presente estudo, cerca de 59 mulheres representando 7,28\% das puérperas afirmaram que houve algum tipo de violência obstétrica no parto. Além disso, o parto violento aumentou o escore de ansiedade-estado. Nesse sentido, destaca-se a violência obstétrica como um tipo específico de violência contra a mulher. O Ministério da Saúde (2012) computou que 12,7\% das queixas das mulheres versavam sobre o tratamento desrespeitoso, incluindo relatos de terem sido mal atendidas, não serem ouvidas ou atendidas em suas necessidades e terem sofrido agressões verbais e físicas (Zanardo, et al., 2017).

No mesmo sentido, Venturini e colaboradores (2010), através da pesquisa "A mulher brasileira nos espaços público e privado", mostraram que $25 \%$ das mulheres entrevistadas relataram ter sofrido algum tipo de violência nos serviços de saúde durante a atenção ao parto, tanto públicos quanto privados (Zanardo, et al., 2017).

Muitas dessas mulheres não têm informação a respeito dos serviços de atendimento, por conta disso elas têm pouca capacidade de ação e decisão. Além disso, muitas não sabem a sua legitimação, o que ocasiona sua subordinação às indicações médicas (García Jordá, et al., 2013).

As mulheres que vivenciam um parto com experiência de ter sofrido uma violência obstétrica como ter sido humilhada pela equipe médica pode desencadear o Transtorno de estresse pós-traumático (TEPT). Os partos em que a mulher vivencia situações como dor intensa e prolongada, experiência de ter sido humilhada pela equipe médica, medo de morte ou morte do feto ou recém-nascido, percepção de anomalia congênita no bebê, entre outras, podem ser considerados fatores traumáticos que eventualmente podem desencadear o TEPT. O DSM-IV define evento traumático como um evento catastrófico envolvendo morte ou lesão real ou ameaçada, ou ameaça à integridade física de si mesma, sendo caracterizada por intenso medo, impotência ou horror (Cantilino, et al., 2010).

Na vertente da humanização o Ministério da Saúde recomenda que para satisfação das mulheres e para usufruto de uma experiência de parto positiva, ao lidar com o manejo do trabalho de parto, "os profissionais de saúde devem refletir como suas próprias crenças e valores influenciam a sua atitude em lidar com a dor do parto e garantir que os seus cuidados apoiem a escolha da mulher" (Silva, 2019).

O tipo de parto, influenciou diretamente na ansiedade das puérperas sendo o parto cesáreo o mais prevalente nos escores altos de ansiedade. A escolha das gestante sobre as vias de parto acontece no pré-natal em que se faz necessário a participação de uma equipe que desempenha atividades e informações em relação ao momento do parto (Costa, et al., 2014).

A respeito do parto normal, é um método que tem diversos benefícios, como sua reparação imediata, se houver complicação elas são menos graves em comparação ao parto cesáreo, a infecção hospitalar é ainda menor e, por fim, a amamentação do recém-nascido se torna mais fácil. Já o parto cesáreo, no Brasil, possui uma taxa elevada de $40 \%$ sendo muito acima do limite de 15\% esperado pela OMS. Esse aumento da frequência de parto cesáreo não apresentou uma associação positiva com o aumento simétrico dos benefícios para a mãe e recém-nascido, fato que corrobora com dados da Organização Mundial de Saúde, que 
caracteriza o parto operatório como uma das práticas mais frequentes, sendo utilizado de modo inadequado e desordenado (Vicente, et al., 2017).

O Coren SP (2010) traz como benefícios do parto natural a possibilidade das gestantes se alimentarem (alimentos leves, que lhes proporcione energia), os riscos de infecções são afastados dessa realidade, não existirá efeitos colaterais de medicamentos e anestésicos, o pós-parto é considerado mais adaptativo não havendo feridas, dor e o cuidado com o recém-nascido é realizado pela própria mãe proporcionando uma melhor interação mãe e filho. Já nessa interação entre mãe e filho, logo com o nascimento o contato com a mãe é imediato, sendo iniciada a primeira mamada com uma maior durabilidade devido ao conforto materno, sem estar sob efeito de medicações a descida do leite materno é mais rápida prevenindo assim a hipoglicemia, diarreias e desidratação; há uma grande diminuição das intervenções que são realizadas no neonato e ainda traz menos riscos de problemas respiratórios (Nascimento, 2016).

Dessa maneira, essa interação mãe e filho com o contato pele a pele e a amamentação na primeira hora pós-parto também foram fatores de uma diminuição no escore de ansiedade no presente estudo. Além disso, esse contato do binômio estimula também os sistemas sensórios do bebê, sendo observado uma redução ou aumento da atividade motora, aumento do movimento ocular e direcionamento da face ao ouvir a voz materna. Enquanto que as mães sentiram vontade de tocar e acariciar o bebê, deixando-os tranquilos (Martins, 2018).

O contato e a amamentação, logo após o parto, são recomendações da Organização Mundial de Saúde (OMS) do Fundo das Nações Unidas para a Infância (UNICEF) e correspondem ao quarto passo da Iniciativa Hospital Amigo da Criança (IHAC). São inúmeros os benefícios imediatos do aleitamento materno exclusivo logo após o parto. Para o recém- nascido, o colostro conhecido como a "primeira vacina" garante a capacidade contra infecções, como por exemplo, para enterocolite necrosante, infecções do trato gastrointestinal e respiratório, alergias, septicemia e meningites, além de estar sempre pronto na temperatura ideal para a criança. Igualmente, o aleitamento materno imediato após o parto é benéfico para a mãe, já que a sucção estimula a liberação de ocitocina endógena, que induz a contração uterina, prevenindo hemorragias puerperais, principal causa de mortalidade materna no mundo (Gayeski \& Brüggemann, 2010).

A posição no período expulsivo afetou ambos os questionários IDATE, sendo mais específica no idate Traço, em que a posição ginecológica demonstrou um maior escore de ansiedade. Desde a década de 70, estudos apontam para a redescoberta das posturas verticais e da mobilidade materna durante o trabalho de parto como prática que volta a mostrar evidências vantajosas e seguras para a parturiente. Na posição vertical há menos irregularidade na contratilidade uterina em sua forma e ritmo, podendo ser consequência da melhor irrigação sanguínea do útero nesta posição, além de prevenir a oclusão da aorta e da veia cava, consequência da postura em decúbito dorsal, assegurando assim o intercâmbio materno-fetal e diminuindo o risco de sofrimento fetal, mesmo num período expulsivo prolongado (Bavaresco, et al., 2011). Além disso, é significante no auxílio respiratório, já que a respiração materna é prejudicada na posição horizontal (Editora Limay, 2017). Porém, a posição supina tem sido adotada com maior frequência no segundo período do trabalho de parto, mesmo havendo evidências que ela ocasione uma redução da eficiência uterina e fluxo sanguíneo placentário (Martins, 2018).

Ter um acompanhante de livre escolha diminuiu o escore de ansiedade no pós parto imediato. Nesse sentido, a violência obstétrica compreende também a proibição do acompanhante mesmo sendo um direito garantido em lei (Lei do Acompanhante: Lei no 11.108/2005) (Zanardo, et al., 2017). De forma semelhante ao acompanhante tem o trabalho das doulas, por exemplo, através desse suporte físico e emocional adequado foi capaz de reduzir o tempo de trabalho de parto, o uso de ocitocina, de analgesia, de parto vaginal operatório e de cesáreas (Haddad \& Cececatti, 2011). Dessa maneira, reduzem também a ansiedade e contribuem de forma positiva no bem estar da mulher. Além disso, compreendendo que o parto é um momento tenso, que pode levar a dor e provocar medo, a presença de um acompanhante de sua confiança faz com que a parturiente se sinta mais segura durante esse período reduzindo a necessidade de medicações para alívio da dor e até mesmo os casos de depressão pós-parto (Cunha, 2014). 
Os métodos não farmacológicos de alívio da dor oferecidos pelo hospital, como exercícios com a bola, banho morno e massagens, contribuíram diminuindo a pontuação no escore de ansiedade.

Durante o trabalho de parto, a água morna reduz a sensibilidade dolorosa da parturiente com redução da atividade simpática através da modificação da transmissão aferente nociceptiva, a qual se torna mais lenta e eleva os níveis de encefalinas e endorfinas endógenas. A utilização do chuveiro, deixando a água cair sobre as costas da gestante, alivia a dor lombar, queixa presente em um terço das parturientes no primeiro estágio do parto, geralmente em consequência da apresentação posterior da cabeça do feto (ODP), provavelmente devido à demora na rotação do pólo cefálico (Bavaresco, et al., 2011).

Outro recurso que pode ser utilizado para a diminuição da ansiedade e sensação dolorosa é a massagem, que vem sendo amplamente recomendada pela Organização Mundial de Saúde. Em um estudo randomizado, realizado com 60 primíparas, que teve como objetivo avaliar o efeito da massagem durante as contrações uterinas, concluiu-se que houve uma redução significativa na intensidade da dor nas duas primeiras fases do trabalho de parto (Martins, 2018). Além disso, a massagem é eficaz no alívio da ansiedade, do estresse e da dor. No entanto, seu efeito em relação à dor, é significativamente maior na fase latente, apesar de também reduzi-la na fase ativa (Gayeski \& Brüggemann, 2010).

$\mathrm{O}$ uso da bola no trabalho de nascimento traz vários benefícios à parturiente, entre eles a correção da postura, o relaxamento e alongamento e o fortalecimento da musculatura. Além disso, os exercícios na bola com a paciente sentada trabalha músculos como o assoalho pélvico, principalmente os músculos levantadores do ânus e pubococcígeo, além da fáscia da pele, o que causa alargamento da pelve ajudando na descida do feto no canal de parto ocasionando benefícios psicológicos com baixo custo financeiro. As principais vantagens dos métodos não farmacológicos são a participação ativa da parturiente e de seu acompanhante durante o parto e nascimento, a não utilização de equipamentos sofisticados e a redução das intervenções (de Oliveira, 2015).

Já a respeito da dieta líquida oferecida no hospital do presente estudo (água ou gelatina), foi um componente de aumento no escore de ansiedade.

É sabido que, diante do alto gasto energético que o trabalho de parto demanda, a parturiente precisa manter uma boa hidratação e aporte calórico, permitindo assim um maior conforto durante o trabalho de parto, por meio da ingestão de alimentos leves e fluidos. Nesse sentido, manter a parturiente em restrição quanto à ingesta hídrica e abstenção de alimentos pode levar a um quadro clínico de cetose e desidratação. O jejum só é necessário se existir a possibilidade do parto evoluir para uma cesariana ou de anestesia geral (Martins, 2018) (Editora Limay, 2017).

\section{Conclusão}

Portanto, os componentes das boas práticas na sala de parto que influenciam diminuindo o escore de ansiedade são contato pele a pele, amamentação na primeira hora pós-parto, parto normal, uso das práticas não farmacológicas para alívio da dor, posição de expulsão, presença do acompanhante de livre escolha e um parto não violento.

O estudo presente proporciona novas pesquisas para o desenvolvimento da temática abordada a fim de promover cada vez mais medidas que confortem as mulheres no momento do parto. Vale ressaltar que novos estudos podem ser realizados a fim de aumentar o número da amostra, seja validando ou confrontando os resultados deste. Além disso, há possibilidade de encontrar novos métodos que não foram descritos e que diminuam também o escore de ansiedade. Por fim, é de suma importância que o tema seja cada vez mais disseminado entre os profissionais de saúde.

\section{Referências}

Barbetta, P. A. (2002). Estatística aplicada às ciências sociais. UFSC Editora. 
Bavaresco, G. Z., Souza, R. S. O. D., Almeica, B., Sabatino, J. H., \& Dias, M. (2011). O fisioterapeuta como profissional de suporte à parturiente. Ciência \& Saúde Coletiva, 16, 3259-3266.

Biaggio, A. M. B., Natalício, L., \& Spielberger, C. D. (1977). Desenvolvimento da forma experimental em português do Inventário de Ansiedade Traço-Estado (IDATE) de Spielberger. Arquivos brasileiros de psicologia aplicada, 29(3), 31-44.

Cantilino, A., Zambaldi, C. F., Sougey, E. B., \& Rennó Jr, J. O. E. L. (2010). Transtornos psiquiátricos no pós-parto. Archives of Clinical Psychiatry (São Paulo), $37,288-294$.

Cechin, P. L. (2002). Reflexões sobre o resgate do parto natural na era da tecnologia. Revista Brasileira de Enfermagem [online], 55(4), 444-448.

Costa, S. P., Prates, R. D. C. G., \& Campelo, B. Q. A. (2014). Parto normal ou cesariana? Fatores que influenciam na escolha da gestante. Revista de Enfermagem da UFSM, 4(1), 1-9.

Cunha, J. F. D. (2014). A perspectiva do pai acompanhante junto à parturiente no momento do parto natural: uma revisão integrativa.

de Almeida, L. W. S., Santos, L. A., de Faria, F. S., de Souza, L. R., Barreto, M. N. L., de Oliveira, Y. N., \& Leite, D. C. F. (2020). Avaliação dos componentes de ansiedade em pacientes no pós-parto imediato em uma maternidade da rede SUS em Aracaju/SE/Evaluation of anxiety components in patients at immediate postpartum period at a SUS maternity hospital in Aracaju/SE. Brazilian Journal of Health Review, 3(6), 18628-18649.

de Freitas Martins, É. (2018). Assistência humanizada às parturientes: análise das práticas desenvolvidas em uma maternidade do sertão paraibano. Fisioterapia Brasil, 19(5), S137-S146.

de Oliveira, L. A. R. (2015). Boas práticas de atenção ao parto: informação e implantação junto a equipe de enfermagem.

Editora Limay (2017). Revista Contemporânea de GO Feminina: Humanização no atendimento ao parto. Autor.

Gallo, R. B. S., Santana, L. S., Marcolin, A. C., Ferreira, C. H. J., Duarte, G., \& Quintana, S. M. (2011). Recursos não-farmacológicos no trabalho de parto: protocolo assistencial. Femina, 41-48.

García Jordá, D., Díaz Bernal, Z., \& Acosta Álamo, M. (2013). El nacimiento en Cuba: análisis de la experiencia del parto medicalizado desde una perspectiva antropológica. Revista Cubana de Salud Pública, 39, 718-732.

Gayeski, M. E., \& Brüggemann, O. M. (2010). Métodos não farmacológicos para alívio da dor no trabalho de parto: uma revisão sistemática. Texto \& ContextoEnfermagem, 19(4), 774-782.

Gonçalves, R., Aguiar, C. A., Merighi, M. A. B. \& Jesus, M. C. P. (2011). Vivenciando o cuidado no contexto de uma casa de parto: o olhar das usuárias. Revista da Escola de Enfermagem da USP, 45(1), 62-70.

Haddad, S. E. M. T., \& Cececatti, J. G. (2011). Estratégias dirigidas aos profissionais para a redução das cesáreas desnecessárias no Brasil. Revista Brasileira de Ginecologia e Obstetrícia, 33, 252-262.

Macedo, P. O., Quitete, J. B., Lima, E. C., dos Santos, I. \& Vargens, O. M. C. (2008). As tecnologias de cuidado de enfermagem obstétricas fundamentais pela teoria ambientalista de Florence Nightingale. Rev. Enferm, 12(8), 341-347.

Matei, E. M., de Carvalho, G. M., Silva, M. B. H. \& Merighi, M. A. B. (2003). Parto humanizado: um direito a ser respeitado. Centro universitário São Camilo, 9(2), 16-23.

Mendes, Y. M. M. B., \& Rattner, D. (2020). Estrutura e práticas de hospitais integrantes do Projeto Apice ON: estudo de linha de base. Revista de Saúde Pública, 54 .

Ministério da Saúde. (2011). Dor no recém-nascido [Versão eletrônica], Atenção à Saúde do Recém-Nascido: Guia para os profissionais de saúde. Intervenções comuns, icterícia e infecções, 2, 33-45.

Moura, E. C. C., Fernandes, M. A., \& Apolinário, F. I. R. (2011). Percepção materna sobre transtornos psiquiátricos no puerpério: implicações na relação mãefilho. Revista Brasileira de Enfermagem, 64, 445-450.

Moura, T. B. D. (2012). Ações de enfermagem para o manejo adequado e humanizado da gestante adolescente primária na admissão e pré-parto do centro obstétrico.

Nascimento, E. S. D. S. (2016). O resgate histórico do nascimento.

Pereira, R. D. R., Franco, S. C., \& Baldin, N. (2011). Representações sociais e decisões das gestantes sobre a parturição: protagonismo das mulheres. Saúde e Sociedade, 20, 579-589.

Silva, M. R. C. D. (2019). Dor e violencia no parto: maus tratos a mulheres em uma maternidade de Manaus.

Souza, F. M. L. C., dos Santos, W. N., Santos, R. S. C., Rodrigues, O. B., Santiago, J. C. D. \& da Silva, R. A. R. (2019). Tecnologias apropriadas ao processo de trabalho de parto humanizado. Enferm Foco, 10(2), 118-124.

Vicente, A. C., Lima, A. K. B. S., \& Lima, C. B. (2017). Parto cesário e parto normal: uma abordagem acerca de riscos e benefícios. Temas em Saúde, 17(4), 24-35.

Zanardo, G. L. D. P., Uribe, M. C., Nadal, A. H. R. D., \& Habigzang, L. F. (2017). Violência obstétrica no Brasil: uma revisão narrativa. Psicologia \& sociedade, 29. 